\title{
Multisensor Estimation Fusion of Nonlinear Cost Functions in Mixed Continuous-Discrete Stochastic Systems
}

\author{
Il Young Song, ${ }^{1}$ Vladimir Shin, ${ }^{2}$ Seokhyoung Lee, ${ }^{3}$ and Won Choi ${ }^{4}$ \\ ${ }^{1}$ Department of Sensor Systems, Hanwha Corporation ReD Center, 52-1 Oesam-dong, Yuseong-gu, \\ Daejeon 305-106, Republic of Korea \\ ${ }^{2}$ Department of Information and Statistics, Research Institute of Natural Science, Gyeongsang National University, \\ 501 Jinjudaero, Gyeongsangnam-do, Jinju 660-701, Republic of Korea \\ ${ }^{3}$ Department of Automation \& Control Research, Hyundai Industrial Research Institute, 1000 Bangeojinsunhwan-doro, \\ Dong-gu, Ulsan 682-792, Republic of Korea \\ ${ }^{4}$ Department of Mathematics, Incheon National University, 119 Academy-ro, Yeonsu-gu, Incheon 406-772, Republic of Korea
}

Correspondence should be addressed to Vladimir Shin; vishin@gnu.ac.kr and Won Choi; choiwon@incheon.ac.kr

Received 27 November 2013; Accepted 26 February 2014; Published 10 April 2014

Academic Editor: Zhengguang Wu

Copyright (C) 2014 Il Young Song et al. This is an open access article distributed under the Creative Commons Attribution License, which permits unrestricted use, distribution, and reproduction in any medium, provided the original work is properly cited.

\begin{abstract}
We propose centralized and distributed fusion algorithms for estimation of nonlinear cost function (NCF) in multisensory mixed continuous-discrete stochastic systems. The NCF represents a nonlinear multivariate functional of state variables. For polynomial NCFs, we propose a closed-form estimation procedure based on recursive formulas for high-order moments for a multivariate normal distribution. In general case, the unscented transformation is used for calculation of nonlinear estimates of a cost functions. To fuse local state estimates, the mixed differential difference equations for error cross-covariance between local estimates are derived. The subsequent application of the proposed fusion estimators for a multisensory environment demonstrates their effectiveness.
\end{abstract}

\section{Introduction}

Multisensor data fusion is typically motivated by reducing the overall redundant information obtained from different sensors, increasing information gain by using multiple sensors, increasing the accuracy, and decreasing the uncertainty of the system. Further, multisensor data fusion can give benefits such as extended temporal and spatial coverage, reduced ambiguity, enhanced spatial resolution, and increased dimensionality of the measurement space. This process has attracted growing interest for potential applications in many fields including guidance, robotics, aerospace, target tracking, signal processing, and control [1-3]. In general, two basic fusion approaches are commonly used to process measured sensor data.

If a central processor receives the measurement data from all local sensors directly and processes them in real time, the correlative result is known as the centralized estimation process. One advantage of the centralized estimation is that it involves minimal information loss. However, the centralized estimation approach has several serious drawbacks, including poor survivability and reliability, as well as heavy communication and computational burdens.

In practice, especially when sensors are dispersed over a wide geographic area, there are limitations on the amount of communications allowed among sensors. Also, sensors are provided with processing capabilities. In this case, a certain amount of computation can be performed at the individual sensors and a compressed version of sensor data can be transmitted to a fusion center where the received information is appropriately combined to yield the global inference. The advantage of the distribution of filters is that the parallel structures would lead to increase of the input data rates and make easy fault detection and isolation. However, the accuracy of the distributed estimators is generally lower than that of the centralized estimator. Recently, various distributed and parallel versions of the standard continuous and discrete Kalman filters have been reported for linear dynamic systems 
within a multisensor environment [1, 2, 4-9]. For nonlinear dynamic state-space models, different variants of suboptimal nonlinear filters, such as the unscented Kalman filter, the extended Kalman filter, and their extensions, are proposed in order to enhance the performance of the nonlinear estimation in multisensory environment [10-14].

However, some applications require the estimation fusion of nonlinear functions of state variables, representing useful information for system control, for example, a quadratic form of a state vector, which can be interpreted as a current distance between targets or as the energy of an object [3]. We refer to the nonlinear function as the nonlinear cost function (NCF). Aside from the aforementioned papers, most of the authors have not focused on the estimation of the NCF, considering instead only a state estimation. To the best of our knowledge, there are no methods reported in the literature for estimation fusion of NCFs in a multisensory environment.

Therefore, in this paper, the estimation fusion problem of NCFs of state variables is considered for mixed continuousdiscrete linear systems under a multisensory environment. The continuous-discrete approach allows system to avoid discretization by propagating the estimate and error covariance between measurements in continuous time using an integration routine such as Runge-Kutta. This approach yields the optimal or suboptimal estimate continuously at all times, including times between the data arrival instants. The advantage of the continuous-discrete estimator over the alternative approaches using system discretization is that, in the former, it is not necessary for the sample times to be equally spaced. This means that the cases of irregular and intermittent measurements are easy to handle.

Therefore, the aim of this paper is to develop fusion estimators for arbitrary NCFs under multisensory environment. Centralized and decentralized estimation fusion algorithms for NCFs are proposed and their accuracies are compared.

This paper is organized as follows. Section 2 presents a statement of the estimation fusion problem for NCFs. In Section 3, the globally optimal centralized estimator is derived. In Section 4, we present the main result pertaining to the distributed estimation of NCFs. Here, the key equations for cross-covariance between the local continuousdiscrete estimators are derived. In Section 5, two computation procedures for calculation of estimates of NCFs and cross-covariance are proposed. The procedures are based on the unscented transformation and recursive formulas for moments of multivariate normal distributions. In Section 6, we study the comparative analysis of the proposed fusion estimators via two theoretical examples. In Section 7, the efficiency of the fusion estimators is studied for the case of an unmanned marine prober system. Finally, we conclude our results in Section 8.

\section{Problem Statement}

The general continuous-discrete Kalman multisensory frame-work involves the estimation of the state of a continuous-time linear dynamic system given discrete measurements

$$
\begin{gathered}
\dot{x}_{t}=F_{t} x_{t}+G_{t} v_{t}, \quad t \geq 0, \\
y_{t_{k}}^{(i)}=H_{t_{k}}^{(i)} x_{t_{k}}+w_{t_{k}}^{(i)}, \quad t_{k+1}>t_{k}>\cdots>t_{0}=0, \\
k=1,2, \ldots ; \quad i=1, \ldots, L,
\end{gathered}
$$

where $x_{t} \in \mathfrak{R}^{n}$ is a state vector, $y_{t_{k}}^{(i)} \in \mathfrak{R}^{m_{i}}$ is a measurement vector from $i$ th sensor $(i=1, \ldots, L), v_{t} \in \Re^{q}$ is a zeromean Gaussian white system noise with intensity $Q_{t}$, that is, $\mathbf{E}\left(v_{t} v_{s}^{T}\right)=Q_{t} \delta_{t-s}, w_{t}^{(i)} \in \mathfrak{R}^{m_{i}}, i=1, \ldots, L$, represent white sequences (measurement errors) $w_{t_{k}}^{(i)} \sim \mathbf{N}\left(0, R_{t_{k}}^{(i)}\right)$, and $\delta_{t}$ is the Dirac delta-function. We assume that the initial state $x_{0} \sim$ $\mathbf{N}\left(\bar{x}_{0}, P_{0}\right)$ system and measurement noises $v_{t}, w_{t_{k}}^{(1)}, \ldots, w_{t_{k}}^{(L)}$ are mutually uncorrelated.

A problem associated with such systems (1) is that of estimation of the nonlinear cost function of the state variables

$$
z_{t}=f\left(x_{t}\right): \mathfrak{R}^{n} \longrightarrow \mathfrak{R}
$$

from the overall noisy sensor measurements

$$
\begin{aligned}
& y_{\left[t_{1}: t_{k}\right]}=\left\{y_{\left[t_{1}: t_{k}\right]}^{(1)}, \ldots, y_{\left[t_{1}: t_{k}\right]}^{(L)}\right\}, \\
& y_{\left[t_{1}: t_{k}\right]}^{(i)}=\left\{y_{t_{1}}^{(i)}, \ldots, y_{t_{k}}^{(i)}\right\}, \\
& i=1, \ldots, L .
\end{aligned}
$$

Typical examples of such NCFs may be an arbitrary quadratic form $f\left(x_{t}\right)=x_{t}^{T} \Omega_{t} x_{t}$ representing an energy-like function of an object or square distance $f\left(x_{t}\right)=d^{2}\left(x_{t}, x_{t}^{0}\right)$ between the current $x_{t}$ and nominal $x_{t}^{0}$ states, respectively.

We propose centralized and distributed estimation fusion algorithms for NCFs in the subsequent sections.

\section{Global Optimal Solution-Centralized Estimator}

In this section, the best global optimal (in mean-square error sense) estimation algorithm for an NCF is derived. In the centralized fusion set-up, a multisensory dynamic system (1) can be reformulated into a composite form

$$
\begin{gathered}
\dot{x}_{t}=F_{t} x_{t}+G_{t} v_{t}, \quad t \geq 0, \\
y_{t_{k}}=H_{t_{k}} x_{t_{k}}+w_{t_{k}}, \quad y_{t_{k}} \in \mathfrak{R}^{m}, \quad m=m_{1}+\cdots+m_{L},
\end{gathered}
$$

where

$$
\begin{gathered}
y_{t_{k}}^{T}=\left[\begin{array}{lll}
y_{t_{k}}^{(1)^{T}} & \cdots & y_{t_{k}}^{(L)^{T}}
\end{array}\right], \quad H_{t_{k}}^{T}=\left[\begin{array}{lll}
H_{t_{k}}^{(1)^{T}} & \cdots & H_{t_{k}}^{(L)^{T}}
\end{array}\right], \\
w_{t_{k}}^{T}=\left[\begin{array}{lll}
w_{t_{k}}^{(1)^{T}} & \cdots & w_{t_{k}}^{(L)^{T}}
\end{array}\right], \quad w_{t_{k}} \sim \mathbf{N}\left(0, R_{t_{k}}\right), \\
R_{t_{k}}=\operatorname{diag}\left\{R_{t_{k}}^{(1)}, \ldots, R_{t_{k}}^{(L)}\right\} .
\end{gathered}
$$


Then, the optimal mean-square estimate $\widehat{x}_{t}^{\mathrm{CF}}=\mathbf{E}\left(x_{t} \mid y_{\left[t_{1}, t_{k}\right]}\right)$ of the state $x_{t}$ based on the overall sensor measurements (3) and error covariance $P_{t}^{\mathrm{CKF}}=\operatorname{cov}\left(e_{t}^{\mathrm{CF}}, e_{t}^{\mathrm{CF}}\right), e_{t}^{\mathrm{CF}}=x_{t}-\widehat{x}_{t}^{\mathrm{CF}}$, are given by the centralized continuous-discrete Kalman filter equations $[15,16]$. We refer to the filter as centralized filter (CF):

Time update between measurements:

$$
\begin{aligned}
& \dot{\hat{x}}_{\tau}^{\mathrm{CF}^{-}}=F_{\tau} \widehat{x}_{\tau}^{\mathrm{CF}^{-}}, \quad t_{k-1} \leq \tau \leq t_{k}, \widehat{x}_{t_{k-1}}^{\mathrm{CF}^{-}}=\widehat{x}_{t_{k-1}}^{\mathrm{CF}}, \\
& \dot{P}_{\tau}^{\mathrm{CF}^{-}}=F_{\tau} P_{\tau}^{\mathrm{CF}^{-}}+P_{\tau}^{\mathrm{CF}^{-}} F_{\tau}^{T}+G_{\tau} Q_{\tau} G_{\tau}^{T}, \quad P_{t_{k-1}}^{\mathrm{CF}^{-}}=P_{t_{k-1}}^{\mathrm{CF}},
\end{aligned}
$$

Measurement update at time $\tau=t_{k}$ :

$$
\begin{aligned}
& \widehat{x}_{t_{k}}^{\mathrm{CF}}=\widehat{x}_{t_{k}}^{\mathrm{CF}^{-}}+K_{t_{k}}^{\mathrm{CF}}\left(y_{t_{k}}-H_{t_{k}} \widehat{x}_{t_{k}}^{\mathrm{CF}^{-}}\right), \quad \widehat{x}_{0}^{\mathrm{CF}-}=\bar{x}_{0}, \\
& K_{t_{k}}^{\mathrm{CF}}=P_{t_{k}}^{\mathrm{CF}^{-}} H_{t_{k}}^{T}\left(H_{t_{k}} P_{t_{k}}^{\mathrm{CF}^{-}} H_{t_{k}}^{T}+R_{t_{k}}\right)^{-1}, \\
& P_{t_{k}}^{\mathrm{CF}}=\left(I_{n}-K_{t_{k}}^{\mathrm{CF}} H_{t_{k}}\right) P_{t_{k}}^{\mathrm{CF}}, \quad P_{0}^{\mathrm{CF}-}=P_{0},
\end{aligned}
$$

where $I_{n} \in \mathfrak{R}^{n \times n}$ is an identity matrix.

Note that, in the absence of measurement $y_{t_{k}}$, the CF includes only time update equations (6a).

Next, the global optimal mean-square estimate of NCF $z_{t}=f\left(x_{t}\right)$ based on the overall sensor measurements (3) also represents a conditional mean; that is,

$$
\widehat{z}_{t}^{\mathrm{opt}}=\mathbf{E}\left(z_{t} \mid y_{\left[t_{1}: t_{k}\right]}\right)=\int f\left(x_{t}\right) p\left(x_{t} \mid y_{\left[t_{1}: t_{k}\right]}\right) d x_{t}
$$

where $p\left(x_{t} \mid y_{\left[t_{1}: t_{k}\right]}\right)=\mathbf{N}\left(\widehat{x}_{t}^{\mathrm{CF}}, P_{t}^{\mathrm{CF}}\right)$ is a conditionally Gaussian probability density function with conditional mean $\widehat{x}_{t}^{\mathrm{CF}}=\mathbf{E}\left(x_{t} \mid y_{\left[t_{1}: t_{k}\right]}\right)$ and covariance $P_{t}^{\mathrm{CF}}$ determined by CF equations (6a) and (6b) for composite linear models (4) and (5), including all sensor measurements.

Thus, estimate (7) represents the optimal minimum mean-square error (MMSE) continuous-discrete estimator

$$
\widehat{z}_{t}^{\mathrm{opt}}=\int f\left(x_{t}\right) \mathbf{N}\left(\widehat{x}_{t}^{\mathrm{CK}}, P_{t}^{\mathrm{CK}}\right) d x_{t}
$$

which depends on the centralized Kalman estimate $\widehat{x}_{t}^{\mathrm{CF}}$ and its error covariance $P_{t}^{\mathrm{CF}}$.

In distributed fusion, the fusion center tries to get the best estimate of an NCF with the processed data received from each local sensor $y_{\left[t_{1}: t_{k}\right]}^{(i)}=\left\{y_{t_{1}}^{(i)}, \ldots, y_{t_{k}}^{(i)}\right\}$. In Sections 4 and 5, we propose the distributed estimation fusion algorithm based on the $L$ local Kalman estimates $\widehat{x}_{t}^{(i)}=\mathbf{E}\left(x_{t} \mid y_{\left[t_{1}: t_{k}\right]}^{(i)}\right)$, $i=1, \ldots, L$, which are available at the fusion center.

\section{Distributed Estimation Fusion Algorithm for Nonlinear Cost Function}

4.1. Local Kalman Estimates. From the local sensor $y_{t_{k}}^{(i)}$, the corresponding local Kalman state estimate $\widehat{x}_{t}^{(i)}=\mathbf{E}\left(x_{t} \mid\right.$ $\left.y_{\left[t_{1}: t_{k}\right]}^{(i)}\right)$ can be calculated using the continuous-discrete Kalman filter equations $[15,16]$. Thus, we have the following:

Time update between measurements:

$$
\begin{aligned}
& \dot{\hat{\dot{x}}}_{\tau}^{(i)^{-}}=F_{\tau} \widehat{x}_{\tau}^{(i)^{-}}, \quad t_{k-1} \leq \tau \leq t_{k}, \widehat{x}_{t_{k-1}}^{(i)^{-}}=\widehat{x}_{t_{k-1}}^{(i)}, \\
& \dot{P}_{\tau}^{(i i)^{-}}=F_{\tau} P_{\tau}^{(i i)^{-}}+P_{\tau}^{(i i)^{-}} F_{\tau}^{T}+G_{\tau} Q_{\tau} G_{\tau}^{T}, \quad P_{t_{k-1}}^{(i i)^{-}}=P_{t_{k-1}}^{(i i)},
\end{aligned}
$$

Measurement update at time $\tau=t_{k}$ :

$$
\begin{aligned}
& \widehat{x}_{t_{k}}^{(i)}=\widehat{x}_{t_{k}}^{(i)^{-}}+K_{t_{k}}^{(i)}\left(y_{t_{k}}^{(i)}-H_{t_{k}}^{(i)} \widehat{x}_{t_{k}}^{(i)^{-}}\right), \quad \widehat{x}_{0}^{(i)}=\bar{x}_{0}, \\
& K_{t_{k}}^{(i)}=P_{t_{k}}^{(i i)^{-}} H_{t_{k}}^{(i)^{T}}\left(H_{t_{k}}^{(i)} P_{t_{k}}^{(i i)^{-}} H_{t_{k}}^{(i)^{T}}+R_{t_{k}}^{(i)}\right)^{-1}, \\
& P_{t_{k}}^{(i i)}=\left(I_{n}-K_{t_{k}}^{(i)} H_{t_{k}}^{(i)}\right) P_{t_{k}}^{(i i)^{-}}, \quad P_{0}^{(i i)-}=P_{0} .
\end{aligned}
$$

Next, we propose suboptimal distributed estimation fusion algorithm based on the local Kalman estimates and error covariance $\left(\widehat{x}_{\tau}^{(i)}, P_{\tau}^{(i i)}\right), P_{\tau}^{(i i)}=\operatorname{cov}\left(e_{\tau}^{(i)}, e_{\tau}^{(i)}\right), i=1, \ldots, L$.

4.2. Distributed Fusion Estimator. The proposed distributed algorithm is comprised of two stages: first, the original local Kalman estimates $\hat{x}_{t}^{(1)}, \ldots, \widehat{x}_{t}^{(L)}$ are transformed to local optimal (in a mean-square sense) nonlinear estimates of an NCF $\widehat{z}_{t}^{(1)}, \ldots, \widehat{z}_{t}^{(L)}$ and, at the second stage, the transformed estimates $\widehat{z}_{t}^{(i)}, i=1, \ldots, L$ are linearly fused based on the fusion formula with scalar weights $[5,6,8]$.

The optimal local mean-square estimate of NCF $z_{t}=$ $f\left(x_{t}\right)$ based on the local sensor measurements $y_{\left[t_{1}: t_{k}\right]}^{(i)}$ represents a conditional mean; that is,

$$
\widehat{z}_{t}^{(i)}=\mathbf{E}\left(z_{t} \mid y_{\left[t_{1}: t_{k}\right]}^{(i)}\right)=\int f\left(x_{t}\right) p\left(x_{t} \mid y_{\left[t_{1}: t_{k}\right]}^{(i)}\right) d x_{t}
$$

where $p\left(x_{t} \mid y_{\left[t_{1}: t_{k}\right]}^{(i)}\right)=\mathbf{N}\left(\widehat{x}_{t}^{(i)}, P_{t}^{(i i)}\right)$ is a local conditional Gaussian density function.

Therefore, the optimal local estimate $\widehat{z}_{t}^{(i)}$ in (10) represents a nonlinear function of the local Kalman estimate and its error covariance; that is, $\widehat{z}_{t}^{(i)}=\widehat{z}_{t}^{(i)}\left(\widehat{x}_{t}^{(i)}, P_{t}^{(i i)}\right)$. Next, using the nonlinear estimates $\widehat{z}_{t_{k}}^{(1)}, \ldots, \widehat{z}_{t_{k}}^{(L)}$ and the fusion formula with scalar weights, we obtain the distributed fusion estimator for an NCF

$$
\widehat{z}_{t}^{\mathrm{fus}}=\sum_{i=1}^{L} a_{t}^{(i)} \widehat{z}_{t}^{(i)}, \quad \sum_{i=1}^{L} a_{t}^{(i)}=1,
$$


where scalar weights $a_{t}^{(i)} \in \mathfrak{R}$ are defined as

$$
\begin{aligned}
& a_{t}=\left[\begin{array}{lll}
a_{t}^{(1)} & \ldots & a_{t}^{(L)}
\end{array}\right] \in \mathfrak{R}^{L}, \\
& a_{t}=\frac{\mathbf{1}_{\mathbf{L}}^{\mathrm{T}} P_{z, t}^{-1}}{\left(\mathbf{1}_{\mathbf{L}}^{\mathrm{T}} P_{t_{k}}^{-1} \mathbf{1}_{\mathbf{L}}\right)}, \quad \mathbf{1}_{\mathbf{L}}=[1 \cdots 1]^{T} \in \mathfrak{R}^{L},
\end{aligned}
$$

where

$$
\begin{array}{r}
P_{z, t}=\left[\operatorname{tr}\left(P_{z, t}^{(i j)}\right)\right]_{i, j=1}^{L} \in \mathfrak{R}^{L \times L}, \\
P_{z, t}^{(i j)}=\operatorname{cov}\left(e_{z, t}^{(i)}, e_{z, t}^{(j)}\right), \\
e_{z, t}^{(i)}=z_{t}-\widehat{z}_{t}^{(i)}=f\left(x_{t}\right)-\widehat{z}_{t}^{(i)}, \\
i, j=1, \ldots, L .
\end{array}
$$

Since the local NCF estimates $\widehat{z}_{t}^{(i)}$ in (10) represent a nonlinear transformation of the local state estimates and their error covariance, $\widehat{z}_{t}^{(i)}=\widehat{z}_{t}^{(i)}\left(\widehat{x}_{t}^{(i)}, P_{t}^{(i i)}\right)$, the cross-covariance $P_{z, t}^{(i j)}$ in (12) depends on the local covariance $P_{t}^{(i i)}=\operatorname{cov}\left(e_{t}^{(i)}, e_{t}^{(i)}\right)$, $i=1, \ldots, L$, determined by the Kalman equations (9a) and (9b), and the local cross-covariance $P_{t}^{(i j)}=\operatorname{cov}\left(e_{t}^{(i)}, e_{t}^{(j)}\right), i \neq j$, which can be described by the equations

Time update between measurements:

$$
\begin{aligned}
& \dot{P}_{\tau}^{(i j)-}=F_{\tau} P_{\tau}^{(i j)-}+P_{\tau}^{(i j)-} F_{\tau}^{T}+G_{\tau} Q_{\tau} G_{\tau}^{T}, \quad t_{k-1} \leq \tau \leq t_{k} \\
& P_{t_{k-1}}^{(i j)-}=P_{t_{k-1}}^{(i j)},
\end{aligned}
$$

Measurement update at time $\tau=t_{k}$ :

$$
\begin{array}{r}
P_{t_{k}}^{(i j)}=\left(I_{n}-K_{t_{k}}^{(i)} H_{t_{k}}^{(i)}\right) P_{t_{k}}^{(i j)-}\left(I_{n}-K_{t_{k}}^{(j)} H_{t_{k}}^{(j)}\right)^{T}, \\
i, j=1, \ldots, L ; \quad i \neq j,
\end{array}
$$

$P_{0}^{(i j)-}=P_{0}$,

where the filter gains $K_{t_{k}}^{(i)}, i=1, \ldots, L$, are determined by (9a) and (9b).

The derivation of (13a) and (13b) is given in the appendix.

\subsection{Discussion}

(1) The local error cross-covariances $P_{t}^{(i j)}, P_{z, t}^{(i j)}$ and weights $a_{t}^{(i)}$ can be precomputed, because they do not depend on the sensor measurements $y_{t_{k}}^{(i)}, i=$ $1, \ldots, L$, but only on the noise statistics $Q_{t}, R_{t_{k}}^{(i)}$, the system matrices $F_{t}, G_{t}, H_{t_{k}}^{(i)}$, the initial conditions $\bar{x}_{0}, P_{0}$, and the NCF $z_{t} \stackrel{=}{=} f\left(x_{t}\right)$, which are the part of system models (1) and (2). Thus, once the measurement schedule has been settled, the real-time implementation of the distributed estimator requires only the computation of the local estimates $\widehat{x}_{t}^{(i)}, \widehat{z}_{t}^{(i)}$ and the final fusion estimate $\widehat{z}_{t}^{\text {fus }}$ of an NCF.
(2) The implementation of the distributed estimator consists of two stages: off-line and on-line. The offline stage is more complex than the off-line stage. This is because it requires the computation of the local cross-covariance and weights. However, it is not essential because this stage can be precomputed. The on-line stage (real-time implementation) requires the computation of only the local and fusion estimates. Therefore, the complexity of the on-line stage is not critical for the distributed estimator. However, to compute $\widehat{z}_{t}^{\text {opt }}$, the centralized estimator requires all sensor measurements together at each time instant $k=1,2, \ldots$, whereas the distributed estimator computes $\widehat{x}_{t}^{(i)}$ and $\widehat{z}_{t}^{(i)}$ sequentially.

In the following, we discuss two computational algorithms for evaluation of fusion estimate (10) depending on the type of NCF.

\section{Numerical Calculation of Estimates of Nonlinear Cost Function}

5.1. Multivariate Polynomial Cost Function Recursive Procedure. Let a special NCF (2) represent an arbitrary multivariate polynomial function of the form

$$
\begin{array}{r}
z=f(x)=\sum_{0 \leq \ell_{1}+\cdots+\ell_{n} \leq n} D_{\ell_{1} \ell_{2} \ldots \ell_{n}} x_{1}^{\ell_{1}} x_{2}^{\ell_{2}}, \ldots, x_{n}^{\ell_{n}}, \\
\ell_{1}, \ldots, \ell_{n} \geq 0 .
\end{array}
$$

Then, the local estimate $\widehat{z}_{t}^{(i)}=\mathbf{E}\left[f\left(x_{t}\right) \mid y_{\left[t_{1}: t_{k}\right]}^{(i)}\right]$ has a closedform solution because conditional expectation $\mathbf{E}\left[f\left(x_{t}\right)\right.$ । $\left.y_{\left[t_{1}: t_{k}\right]}^{(i)}\right]$ and cross-covariance $P_{z, t}^{(i j)}$ depend on high-order moments $\widehat{m}_{\ell_{1} \ell_{2} \cdots \ell_{n}} \equiv \mathbf{E}\left(x_{1}^{\ell_{1}} x_{2}^{\ell_{2}}, \ldots, x_{n}^{\ell_{n}} \mid y_{\left[t_{1}: t_{k}\right]}^{(i)}\right)$ or $m_{\ell_{1} \ell_{2}, \ldots, \ell_{n}} \equiv$ $\mathbf{E}\left(x_{1}^{\ell_{1}} x_{2}^{\ell_{2}} \cdots x_{n}^{\ell_{n}}\right)$ of a multivariate Gaussian distribution, which can be calculated explicitly in terms of first- and second-order moments $\hat{x}_{t}^{(i)}=\mathbf{E}\left(x_{t} \mid y_{\left[t_{1}: t_{k}\right]}^{(i)}\right)$ and $P_{t}^{(i j)}, i, j=$ $1, \ldots, n$, using recursive formulas [17-19]. For example,

$$
\begin{aligned}
m_{\ell_{1} \ell_{2}, \ldots, \ell_{n}}= & \sum_{i=2}^{n} \ell_{i} P_{t_{k}}^{(1 i)} m_{\ell_{1}-1, \ldots, \ell_{i}-1, \ldots, \ell_{n}} \\
& +\left(\ell_{1}-1\right) P_{t_{k}}^{(11)} m_{\ell_{1}-2, \ell_{2}, \ldots, \ell_{n}}
\end{aligned}
$$

with the first term vanishing when $\ell_{1}=1$ [19].

The following example illustrates the closed-form computational procedure.

Consider an arbitrary quadratic cost function

$$
z_{t}=f\left(x_{t}\right)=x_{t}^{T} \Omega_{t} x_{t}, \quad \Omega_{t}^{T}=\Omega_{t}, \quad \Omega_{t}>0 .
$$

Show that the optimal local estimate $\widehat{z}_{t}^{(i)}$ can be calculated explicitly in terms of a local state estimate and its error covariance. Using formula $\mathrm{E}\left(x^{T} \Omega x\right)=\operatorname{tr}\left[\Omega\left(P+m m^{T}\right)\right]$, $m=\mathbf{E}(x), P=\operatorname{cov}(x, x)$ [17], we obtain an optimal local estimate for the quadratic cost function

$$
\widehat{z}_{t}^{(i)}=\mathbf{E}\left(x_{t}^{T} \Omega_{t} x_{t} \mid y_{\left[t_{1}: t_{k}\right]}^{(i)}\right)=\operatorname{tr}\left\{\Omega_{t}\left(P_{t}^{(i i)}+\widehat{x}_{t}^{(i)} \widehat{x}_{t}^{(i)^{T}}\right)\right\},
$$


where the local Kalman estimate and error covariance $\left(\widehat{x}_{t}^{(i)}, P_{t}^{(i i)}\right)$ satisfy $(9 \mathrm{a})$ and $(9 \mathrm{~b})$.

5.2. General Cost Function and Unscented Transformation. During the last decade, the unscented transformation (UT) has become a powerful approach for designing computationally effective algorithms for nonlinear models [10-12, 14, 20]. Following this, the procedure to calculate the best local estimate of an NCF (conditional mean)

$$
\widehat{z}_{t}^{(i)}=\mathbf{E}\left[f\left(x_{t}\right) \mid y_{\left[t_{1}: t_{k}\right]}^{(i)}\right]
$$

using the UT can be summarized as follows.

Generate the sigma points $\left\{X_{s, t}\right\}_{s=0}^{2 n}$ with corresponding weights $\left\{W_{s}\right\}_{s=0}^{2 n}$ :

$$
\begin{gathered}
X_{0, t}^{(i)}=\widehat{x}_{t}^{(i)}, \quad W_{0}=\frac{\ell}{n+\ell}, \\
X_{s, t}^{(i)}=\widehat{x}_{t}^{(i)}+\left[\sqrt{(n+\ell) P_{t}^{(i i)}}\right]_{s}, \quad W_{s}=\frac{1}{2(n+\ell)}, \\
s=1, \ldots, n, \\
X_{s+n, t}^{(i)}=\widehat{x}_{t}^{(i)}-\left[\sqrt{(n+\ell) P_{t}^{(i i)}}\right]_{s}, \quad W_{s+n}=\frac{1}{2(n+\ell)},
\end{gathered}
$$

where $\left[\sqrt{P_{t}^{(i i)}}\right]$ is the sth column of the matrix square root of $P_{t}^{(i i)}$ and $\ell$ is the scaling parameter influencing the spread of points in the state space and thus the accuracy of the approximation [20]. Propagate each of these sigma points through the nonlinear function as

$$
\xi_{s, t}^{(i)}=f\left(X_{s, t}^{(i)}\right), \quad s=0,1, \ldots, 2 n
$$

and the resulting best local estimate of the NCF is given as

$$
\widehat{z}_{t}^{(i)}=\sum_{s=0}^{2 n} W_{s} \xi_{s, t}^{(i)}, \quad i=1, \ldots, L .
$$

Similar to (19)-(21), the local cross-covariance $P_{z, t}^{(i j)}$ in (12) can be calculated based on the UT. But, in a special case of a polynomial NCF (14), they are calculated for a multivariate Gaussian distribution of a composite random vector $U_{t}^{T}=$ $\left[\begin{array}{lll}x_{t}^{T} & \widehat{x}_{t}^{(i)^{T}} & \widehat{x}_{t}^{(j)^{T}}\end{array}\right]$ via the recursive formulas (15).

The best way to gain some insight into the proposed centralized and distributed estimators is to look at some theoretical examples. The comparison analysis of the proposed estimators will be demonstrated in the next section.

\section{Theoretical Comparison of Estimators}

6.1. Example 1: Estimation of Power of a Constant Scalar Unknown. Consider a simple example of an application of the obtained results. We estimate the quadratic cost function $z=\theta^{2}$ of a random constant $\theta \sim \mathbf{N}\left(0, \sigma_{\theta}^{2}\right)$, given two multiple discrete sensor measurements $y_{t_{k}}^{(1)}$ and $y_{t_{k}}^{(2)}$ of $\theta$ corrupted by uncorrelated Gaussian white noises. The mixed continuousdiscrete model describing this situation is

$$
\begin{array}{lll}
\text { System: } & \dot{x}_{t}=0, \quad t \geq 0, & x_{0} \equiv \theta \sim \mathbf{N}\left(0, \sigma_{\theta}^{2}\right), \\
\text { Sensor 1: } & y_{t_{k}}^{(1)}=x_{t_{k}}+w_{t_{k}}^{(1)}, & w_{t_{k}}^{(1)} \sim \mathbf{N}\left(0, r_{1}\right), \\
\text { Sensor 2: } & y_{t_{k}}^{(2)}=x_{t_{k}}+w_{t_{k}}^{(2)}, & w_{t_{k}}^{(2)} \sim \mathbf{N}\left(0, r_{2}\right) .
\end{array}
$$

Here, we derive precise equations for the MSEs for the proposed fusion estimators and demonstrate a comparative analysis.

6.1.1. Centralized Optimal Estimate of Quadratic Cost Function, $\widehat{z}_{t}^{\text {opt }}$. Using (17) at $\Omega_{t}=1$, the global optimal estimate of the quadratic cost function takes the form

$$
\begin{aligned}
\widehat{z}_{\tau}^{\text {opt }} & =\mathbf{E}\left(\theta^{2} \mid y_{\left[t_{1}: t_{k}\right]}\right) \\
& =\int \theta^{2} \mathbf{N}\left(\widehat{\theta}_{\tau}^{\mathrm{CF}}, P_{\tau}^{\mathrm{CF}}\right) d \theta=P_{\tau}^{\mathrm{CF}}+\left(\widehat{\theta}_{\tau}^{\mathrm{CF}}\right)^{2},
\end{aligned}
$$

where $\widehat{\theta}_{\tau}^{\mathrm{CF}} \equiv \widehat{x}_{\tau}^{\mathrm{CF}}=\mathrm{E}\left(x_{\tau} \mid y_{\left[t_{1}: t_{k}\right]}\right)$ is the best global MMSE estimate of an unknown state $x_{t}=\theta$ based on the overall sensor measurements $y_{\left[t_{1}: t_{k}\right]}=\left\{y_{\left[t_{1}: t_{k}\right]}^{(1)}, y_{\left[t_{1}: t_{k}\right]}^{(2)}\right\}$ and $P_{\tau}^{C F}=$ $\mathbf{E}\left[\left(\theta-\widehat{\theta}_{\tau}^{\mathrm{CF}}\right)^{2}\right]$ is its error variance. Using the continuousdiscrete Kalman filter equations (6a) and (6b), we get

Time update between measurements:

$\dot{\hat{\theta}}_{\tau}^{\mathrm{CF}^{-}}=0, \quad t_{k-1} \leq \tau \leq t_{k}, \quad \widehat{\theta}_{t_{k-1}}^{\mathrm{CF}^{-}}=\widehat{\theta}_{t_{k-1}}^{\mathrm{CF}}$,

$\dot{P}_{\tau}^{\mathrm{CF}^{-}}=0, \quad P_{t_{k-1}}^{\mathrm{CF}^{-}}=P_{t_{k-1}}^{\mathrm{CF}}$,

Measurement update at time $\tau=t_{k}$ :

$\widehat{\theta}_{t_{k}}^{\mathrm{CF}}=\widehat{\theta}_{t_{k}}^{\mathrm{CF}-}+K_{t_{k}}^{(1)}\left(y_{t_{k}}^{(1)}-\widehat{\theta}_{t_{k}}^{\mathrm{CF}-}\right)+K_{t_{k}}^{(2)}\left(y_{t_{k}}^{(2)}-\widehat{\theta}_{t_{k}}^{\mathrm{CF}-}\right)$,

$\widehat{\theta}_{0}^{\mathrm{CF}-}=0$,

$K_{t_{k}}^{(1)}=\frac{r_{2} P_{t_{k}}^{\mathrm{CF}-}}{r_{1} r_{2}+\left(r_{1}+r_{2}\right) P_{t_{k}}^{\mathrm{CF}-}}$,

$K_{t_{k}}^{(2)}=\frac{r_{1} P_{t_{k}}^{\mathrm{CF}-}}{r_{1} r_{2}+\left(r_{1}+r_{2}\right) P_{t_{k}}^{\mathrm{CF}-}}$

$P_{t_{k}}^{\mathrm{CF}}=\left(1-K_{t_{k}}^{(1)}-K_{t_{k}}^{(2)}\right) P_{t_{k}}^{\mathrm{CF}-}, \quad P_{0}^{\mathrm{CF}-}=\sigma_{\theta}^{2}$.

Using induction, we obtain the exact formula for the MSE

$$
P_{\tau}^{\mathrm{CF}}=\mathbf{E}\left[\left(\theta-\widehat{\theta}_{\tau}^{\mathrm{CF}}\right)^{2}\right]= \begin{cases}P_{t_{k-1}}^{\mathrm{CF}}, & t_{k-1} \leq \tau<t_{k}, \\ P_{t_{k}}^{\mathrm{CF}}, & \tau=t_{k},\end{cases}
$$

where

$$
P_{t_{k}}^{\mathrm{CF}}=\frac{r \sigma_{\theta}^{2}}{r+k \sigma_{\theta}^{2}}, \quad r=\frac{r_{1} r_{2}}{r_{1}+r_{2}}, \quad k=0,1,2, \ldots
$$


The estimation accuracy between the unknown power $z=\theta^{2}$ and its global fusion estimate

$$
\widehat{z}_{\tau}^{\mathrm{opt}}= \begin{cases}P_{t_{k-1}}^{\mathrm{CF}}+\left(\widehat{\theta}_{t_{k-1}}^{\mathrm{CF}}\right)^{2}, & t_{k-1} \leq \tau<t_{k} \\ P_{t_{k}}^{\mathrm{CF}}+\left(\widehat{\theta}_{t_{k}}^{\mathrm{CF}}\right)^{2}, & \tau=t_{k}\end{cases}
$$

also can be measured in terms of the MSE $P_{\tau}^{\text {opt }}=\mathbf{E}\left[\left(\theta^{2}-\right.\right.$ $\left.\left.\widehat{z}_{\tau}^{\text {opt }}\right)^{2}\right]$. We have

$$
\begin{aligned}
& P_{\tau}^{\mathrm{opt}}= \mathbf{E}\left[\left(\theta^{2}-P_{\tau}^{\mathrm{CF}}-\left(\widehat{\theta}_{\tau}^{\mathrm{CF}}\right)\right)^{2}\right] \\
&= \mathbf{E}\left(\theta^{4}\right)+\left(P_{\tau}^{\mathrm{CF}}\right)^{2}+\mathbf{E}\left[\left(\widehat{\theta}_{\tau}^{\mathrm{CF}}\right)^{4}\right]-2 P_{\tau}^{\mathrm{CF}} \mathbf{E}\left(\theta^{2}\right) \\
&-2 \mathbf{E}\left[\theta^{2}\left(\widehat{\theta}_{\tau}^{\mathrm{CF}}\right)^{2}\right]+2 P_{\tau}^{\mathrm{CF}} \mathbf{E}\left[\left(\widehat{\theta}_{\tau}^{\mathrm{CF}}\right)^{2}\right], \\
& t_{k-1} \leq \tau \leq t_{k} .
\end{aligned}
$$

Using the orthogonality property of the unbiased estimate $\widehat{\theta}_{\tau}^{\mathrm{CF}}$ and the formulas for the fourth-order moments of a bivariate Gaussian random vector $\left[\begin{array}{ll}\theta & \widehat{\theta}_{\tau}^{\mathrm{CF}}\end{array}\right]^{T}$,

$$
\begin{aligned}
& \mathbf{E}\left(\theta^{4}\right)=3\left(\sigma_{\theta}^{2}\right)^{2}, \quad \mathbf{E}\left[\left(\widehat{\theta}_{\tau}^{\mathrm{CF}}\right)^{4}\right]=3\left[\operatorname{Var}\left(\widehat{\theta}_{\tau}^{\mathrm{CF}}\right)\right]^{2}, \\
& \mathbf{E}\left[\theta^{2}\left(\widehat{\theta}_{\tau}^{\mathrm{CF}}\right)^{2}\right]=\sigma_{\theta}^{2} \operatorname{Var}\left(\widehat{\theta}_{\tau}^{\mathrm{CF}}\right)+2\left[\operatorname{cov}\left(\theta, \widehat{\theta}_{\tau}^{\mathrm{CF}}\right)\right]^{2},
\end{aligned}
$$

where

$$
\operatorname{Var}\left(\widehat{\theta}_{\tau}^{\mathrm{CF}}\right)=\operatorname{cov}\left(\theta, \widehat{\theta}_{\tau}^{\mathrm{CF}}\right)=\sigma_{\theta}^{2}-P_{\tau}^{\mathrm{CF}}
$$

we obtain

$$
P_{\tau}^{\mathrm{opt}}=2 P_{\tau}^{\mathrm{CF}}\left(2 \sigma_{\theta}^{2}-P_{\tau}^{\mathrm{CF}}\right), \quad t_{k-1} \leq \tau \leq t_{k} .
$$

Taking into account (25), we get the exact MMSE for the centralized estimator; that is,

$$
P_{\tau}^{\mathrm{opt}}=\mathbf{E}\left[\left(\theta^{2}-\widehat{z}_{\tau}^{\mathrm{CF}}\right)^{2}\right]= \begin{cases}P_{t_{k-1}}^{\mathrm{opt}}, & t_{k-1} \leq \tau<t_{k}, \\ P_{t_{k}}^{\mathrm{opt}}, & \tau=t_{k},\end{cases}
$$

where

$$
\begin{array}{r}
P_{t_{k}}^{\mathrm{opt}}=2 P_{t_{k}}^{\mathrm{CF}}\left(2 \sigma_{\theta}^{2}-P_{t_{k}}^{\mathrm{CF}}\right)=\frac{2 r \sigma_{\theta}^{4}\left(r+2 k \sigma_{\theta}^{2}\right)}{\left(r+k \sigma_{\theta}^{2}\right)}, \quad r=\frac{r_{1} r_{2}}{r_{1}+r_{2}}, \\
k=0,1,2, \ldots
\end{array}
$$

Together with the centralized estimator (26), we apply the distributed estimator developed in Section 4.
6.1.2. Distributed Fusion Estimate, $\widehat{z}_{t}^{\text {fus }}$. Using (9a) and (9b) and (13a) and (13b), the local estimates $\widehat{\theta}_{\tau}^{(i)}=\mathbf{E}\left(x_{\tau} \mid y_{\left[t_{1}: t_{k}\right]}^{(i)}\right)$, error variances $P_{\tau}^{(i i)}=\mathbf{E}\left(e_{\tau}^{(i)^{2}}\right)$, and cross-covariance $P_{\tau}^{(12)}=$ $\mathbf{E}\left(e_{\tau}^{(1)} e_{\tau}^{(2)}\right), e_{\tau}^{(i)}=\theta-\widehat{\theta}_{\tau}^{(i)}, i=1,2$, are described by the following equations:

Time update between measurements:

$$
\begin{aligned}
& \dot{\hat{\theta}}_{\tau}^{(i)^{-}}=0, \quad t_{k-1} \leq \tau \leq t_{k}, \quad \widehat{\theta}_{t_{k-1}}^{(i)^{-}}=\widehat{\theta}_{t_{k-1}}^{(i)}, \\
& \dot{P}_{\tau}^{(i i)^{-}}=0, \quad P_{t_{k-1}}^{(i i)^{-}}=P_{t_{k-1}}^{(i i)}, \quad i=1,2, \\
& \dot{P}_{\tau}^{(12)-}=0, \quad t_{k-1} \leq \tau \leq t_{k}, \quad P_{t_{k-1}}^{(12)-}=P_{t_{k-1}}^{(12)},
\end{aligned}
$$

Measurement update at time $\tau=t_{k}$ :

$$
\begin{aligned}
& \widehat{\theta}_{t_{k}}^{(i)}=\widehat{\theta}_{t_{k}}^{(i)-}+K_{t_{k}}^{(i)}\left(y_{t_{k}}^{(i)}-\widehat{\theta}_{t_{k}}^{(i)-}\right), \quad \widehat{\theta}_{0}^{(i)-}=0, \\
& K_{t_{k}}^{(i)}=\frac{P_{t_{k}}^{(i i)-}}{r_{i}+P_{t_{k}}^{(i i)-}}, \\
& P_{t_{k}}^{(i i)}=\left(1-K_{t_{k}}^{(i)}\right) P_{t_{k}}^{(i i)-}, \quad P_{0}^{(i i)}=\sigma_{\theta}^{2}, \\
& P_{t_{k}}^{(12)}=\left(1-K_{t_{k}}^{(1)}\right)\left(1-K_{t_{k}}^{(2)}\right) P_{t_{k}}^{(12)-}, \quad P_{0}^{(12)}=\sigma_{\theta}^{2} .
\end{aligned}
$$

The solution of (31a) and (31b) is given by

$$
\begin{aligned}
& P_{\tau}^{(i i)}= \begin{cases}P_{t_{k-1}}^{(i i)}, & t_{k-1} \leq \tau<t_{k} \\
P_{t_{k}}^{(i i)}, & \tau=t_{k}\end{cases} \\
& P_{\tau}^{(12)}= \begin{cases}P_{t_{k-1}}^{(12)}, & t_{k-1} \leq \tau<t_{k} \\
P_{t_{k}}^{(12)}, & \tau=t_{k},\end{cases}
\end{aligned}
$$

where

$$
\begin{aligned}
& P_{t_{k}}^{(i i)}=\frac{r_{i} \sigma_{\theta}^{2}}{r_{i}+k \sigma_{\theta}^{2}}, \quad i=1,2, \\
& P_{t_{k}}^{(12)}=\frac{r_{1} r_{2} \sigma_{\theta}^{2}}{\left(r_{1}+k \sigma_{\theta}^{2}\right)\left(r_{2}+k \sigma_{\theta}^{2}\right)}, \quad k=0,1,2, \ldots
\end{aligned}
$$

Next, using formula (10), one can obtain two local estimates for the quadratic cost as $\widehat{z}_{\tau}^{(i)}=P_{\tau}^{(i i)}+\left(\widehat{\theta}_{\tau}^{(i)}\right)^{2}, i=1,2$, where $\widehat{\theta}_{\tau}^{(1)}$ and $\hat{\theta}_{\tau}^{(2)}$ are calculated by (31a) and (31b). In the second stage, 
using fusion formulas (11) and (12), we obtain the distributed fusion estimate

$$
\begin{array}{r}
\widehat{z}_{\tau}^{\text {fus }}=a_{\tau}^{(1)} \widehat{z}_{t_{k}}^{(1)}+a_{\tau}^{(2)} \widehat{z}_{\tau}^{(2)}, \quad a_{\tau}^{(1)}+a_{\tau}^{(2)}=1, \\
t_{k-1} \leq \tau \leq t_{k},
\end{array}
$$

where

$$
\begin{aligned}
& a_{\tau}^{(1)}=\frac{P_{z, \tau}^{(22)}-P_{z, \tau}^{(12)}}{P_{z, \tau}^{(11)}-2 P_{z, \tau}^{(12)}+P_{z, \tau}^{(22)}}, \\
& a_{\tau}^{(2)}=\frac{P_{z, \tau}^{(11)}-P_{z, \tau}^{(12)}}{P_{z, \tau}^{(11)}-2 P_{z, \tau}^{(12)}+P_{z, \tau}^{(22)}}, \\
& P_{z, \tau}^{(i j)}=\operatorname{cov}\left(e_{z, \tau}^{(i)}, e_{z, \tau}^{(j)}\right), \quad e_{z, \tau}^{(i)}=\theta^{2}-\widehat{z}_{\tau}^{(i)}, \quad i, j=1,2 .
\end{aligned}
$$

Calculating the cross-covariance $P_{z, \tau}^{(i j)}$ based on the formulas for high-order moments of a Gaussian distribution (28), we get

$$
\begin{aligned}
P_{z, \tau}^{(i i)}= & \mathbf{E}\left[\left(\theta^{2}-\widehat{z}_{\tau}^{(i)}\right)^{2}\right]=2 P_{\tau}^{(i i)}\left(2 \sigma_{\theta}^{2}-P_{\tau}^{(i i)}\right), \quad i=1,2, \\
P_{z, \tau}^{(12)}= & \mathbf{E}\left[\left(\theta^{2}-\widehat{z}_{\tau}^{(1)}\right)\left(\theta^{2}-\widehat{z}_{\tau}^{(2)}\right)\right]=2 P_{\tau}^{(12)} \\
& +4\left(\sigma_{\theta}^{2} P_{\tau}^{(12)}-P_{\tau}^{(11)} P_{\tau}^{(12)}-P_{\tau}^{(22)} P_{\tau}^{(12)}+P_{\tau}^{(11)} P_{\tau}^{(22)}\right) .
\end{aligned}
$$

Finally, the overall MSE $P_{\tau}^{\text {fus }}=\mathbf{E}\left[\left(\theta^{2}-\widehat{z}_{\tau}^{\text {fus }}\right)^{2}\right]$ for the fusion estimate $\widehat{z}_{\tau}^{\text {fus }}$ can be evaluated as

$$
P_{\tau}^{\text {fus }}=\mathbf{E}\left[\left(\theta^{2}-\widehat{z}_{\tau}^{\text {fus }}\right)^{2}\right]= \begin{cases}P_{t_{k-1}}^{\mathrm{fus}}, & t_{k-1} \leq \tau<t_{k}, \\ P_{t_{k}}^{\mathrm{fus}}, & \tau=t_{k},\end{cases}
$$

where

$$
\begin{array}{r}
P_{t_{k}}^{\text {fus }}=\left(a_{t_{k}}^{(1)}\right)^{2} P_{z, t_{k}}^{(11)}+\left(a_{t_{k}}^{(2)}\right)^{2} P_{z, t_{k}}^{(22)}+2 a_{t_{k}}^{(1)} a_{t_{k}}^{(2)} P_{z, t_{k}}^{(12)}, \\
k=0,1,2, \ldots .
\end{array}
$$

Here, the scalar weights $a_{t_{k}}^{(1)}$ and $a_{t_{k}}^{(2)}$ and cross-covariance $P_{z, t_{k}}^{(i j)}, i, j=1,2$, are determined by (32)-(34) at $\tau=t_{k}$, $k=1,2, \ldots$

\subsubsection{Comparative Analysis of Centralized and Distributed} Estimators. The MSE is an important value that can be used to reflect the accuracy of NCF estimation. The exact MSEs $P_{t}^{\text {opt }}$ and $P_{t}^{\text {fus }}$ are illustrated in Figure 1 for $\sigma_{\theta}^{2}=$ $1, r_{1}=2, r_{2}=3$. Not surprisingly, Figure 1 illustrates that the centralized estimator exhibits a performance that is completely superior to the distributed estimator; that is, $P_{t}^{\mathrm{opt}}<P_{t}^{\text {fus }}$. From Figure 1, we also observe that the difference between two fusion estimators is negligible for steady-state regimes $k \gg 1$. Thus, for the example, application of the distributed estimator can produce good results in real-time processing requirements.

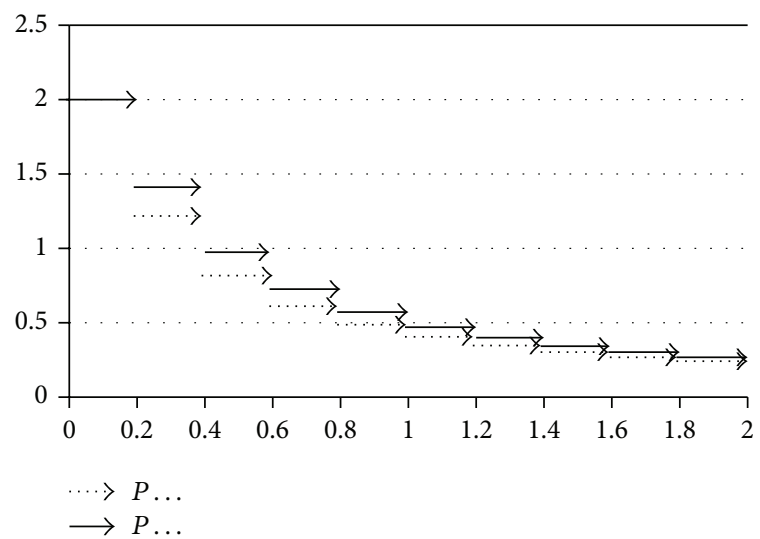

FIGURE 1: MSEs of fusion estimators for quadratic cost function $z=$ $\theta^{2}$.

6.2. Example 2: Estimation of Power of a Scalar Signal. Let the scalar signal $x_{t}$ with two sensors be described by

$$
\begin{gathered}
\dot{x}_{t}=a x_{t}+v_{t}, \quad a<0, t \in\left[0, T_{k}\right], \\
y_{t_{k}}^{(i)}=x_{t_{k}}^{(i)}+w_{t_{k}}^{(i)}, \quad i=1,2,
\end{gathered}
$$

where $v_{t}$ is zero-mean white Gaussian noise with intensity $q$ and $w_{t_{k}}^{(1)} \sim \mathbf{N}\left(0, r_{1}\right)$ and $w_{t_{k}}^{(2)} \sim \mathbf{N}\left(0, r_{2}\right)$ are uncorrelated white Gaussian sequences. Let $x_{0} \sim \mathbf{N}\left(\bar{x}_{0}, \sigma_{0}^{2}\right)$, and an NCF represents power of the signal; that is, $z_{t}=f\left(x_{t}\right)=x_{t}^{2}$.

In a similar way as in Example 1, we can derive equations for MSEs for the proposed estimators.

6.2.1. Centralized Optimal Estimate of Quadratic Cost Function, $\widehat{z}_{t}^{o p t}$. The global MMSE fusion estimate of the power of signal takes the form

$$
\begin{aligned}
& \widehat{z}_{\tau}^{\mathrm{opt}}= \mathbf{E}\left(x_{\tau}^{2} \mid y_{\left[t_{1}: t_{k}\right]}\right) \\
&=\int x^{2} \mathbf{N}\left(\widehat{x}_{\tau}^{\mathrm{CF}}, P_{\tau}^{\mathrm{CF}}\right) d x=P_{\tau}^{\mathrm{CF}}+\left(\widehat{x}_{\tau}^{\mathrm{CF}}\right)^{2}, \\
& t_{k-1} \leq \tau \leq t_{k},
\end{aligned}
$$

where the estimate $\widehat{x}_{\tau}^{\mathrm{CF}}$ and its error variance $P_{\tau}^{\mathrm{CF}}$ are described by the continuous-discrete Kalman filter equations (6a) and (6b)

Time update between measurements:

$$
\begin{aligned}
& \dot{\hat{x}}_{\tau}^{\mathrm{CF}^{-}}=a \widehat{x}_{\tau}^{\mathrm{CF}-}, \quad t_{k-1} \leq \tau \leq t_{k}, \quad \widehat{x}_{t_{k-1}}^{\mathrm{CF}^{-}}=\widehat{x}_{t_{k-1}}^{\mathrm{CF}}, \\
& \dot{P}_{\tau}^{\mathrm{CF}^{-}}=2 a P_{\tau}^{\mathrm{CF}-}+q, \quad P_{t_{k-1}}^{\mathrm{CF}^{-}}=P_{t_{k-1}}^{\mathrm{CF}},
\end{aligned}
$$


Measurement update at time $\tau=t_{k}$ :

$$
\begin{aligned}
& \widehat{x}_{t_{k}}^{\mathrm{CF}}=\widehat{x}_{t_{k}}^{\mathrm{CF}-}+K_{t_{k}}^{(1)}\left(y_{t_{k}}^{(1)}-\widehat{x}_{t_{k}}^{\mathrm{CF}-}\right)+K_{t_{k}}^{(2)}\left(y_{t_{k}}^{(2)}-\widehat{x}_{t_{k}}^{\mathrm{CF}-}\right), \\
& \widehat{x}_{0}^{\mathrm{CF}-}=\bar{x}_{0}, \\
& K_{t_{k}}^{(1)}=\frac{r_{2} P_{t_{k}}^{\mathrm{CF}-}}{r_{1} r_{2}+\left(r_{1}+r_{2}\right) P_{t_{k}}^{\mathrm{CF}-}}, \\
& K_{t_{k}}^{(2)}=\frac{r_{1} P_{t_{k}}^{\mathrm{CF}-}}{r_{1} r_{2}+\left(r_{1}+r_{2}\right) P_{t_{k}}^{\mathrm{CF}-}}, \\
& P_{t_{k}}^{\mathrm{CF}}=\left(1-K_{t_{k}}^{(1)}-K_{t_{k}}^{(2)}\right) P_{t_{k}}^{\mathrm{CF}-}, \quad P_{0}^{\mathrm{CF}-}=\sigma_{0}^{2} .
\end{aligned}
$$

Solving (38a) and (38b) for the error variance, we get

$$
\begin{gathered}
P_{\tau}^{\mathrm{CF}-}=\left(P_{t_{k-1}}^{\mathrm{CF}}+\frac{q}{2 a}\right) e^{2 a\left(\tau-t_{k-1}\right)}-\frac{q}{2 a}, \quad t_{k-1} \leq \tau \leq t_{k}, \\
P_{t_{k}}^{\mathrm{CF}}=\frac{r_{1} r_{2} P_{t_{k}}^{\mathrm{CF}-}}{r_{1} r_{2}+\left(r_{1}+r_{2}\right) P_{t_{k}}^{\mathrm{CF}-}}, \quad k=0,1, \ldots ; \quad P_{0}^{\mathrm{CF}-}=\sigma_{0}^{2} .
\end{gathered}
$$

To find the overall MSE $P_{\tau}^{\text {opt }}=\mathbf{E}\left[\left(x_{\tau}^{2}-\widehat{z}_{\tau}^{\text {opt }}\right)^{2}\right]$, we use the same way as in the derivation of formula (29). We obtain

$$
\begin{aligned}
& P_{\tau}^{\mathrm{opt}}=2 P_{\tau}^{\mathrm{CF}}\left(2 \alpha_{2, \tau}-P_{\tau}^{\mathrm{CF}}\right), \quad \alpha_{2, \tau}=\mathbf{E}\left(x_{\tau}^{2}\right), \\
& t_{k-1} \leq \tau \leq t_{k}
\end{aligned}
$$

where the second-order moment of the signal $\alpha_{2, \tau}$ satisfies the Lyapunov equation

$$
\dot{\alpha}_{2, \tau}=2 a \alpha_{2, \tau}+q, \quad \tau \geq 0, \quad \alpha_{2,0}=\mathbf{E}\left(x_{0}^{2}\right)=\sigma_{0}^{2}+\bar{x}_{0}^{2} .
$$

Finally, using relation (40) between $P_{\tau}^{\mathrm{CF}}$ and $P_{\tau}^{\text {opt }}$, we get

$$
P_{\tau}^{\mathrm{opt}}= \begin{cases}2 P_{\tau}^{\mathrm{CF}}\left(2 \alpha_{2, \tau}-P_{\tau}^{\mathrm{CF}}\right), & t_{k-1} \leq \tau<t_{k}, \\ 2 P_{t_{k}}^{\mathrm{CF}}\left(2 \alpha_{2, t_{k}}-P_{t_{k}}^{\mathrm{CF}}\right), & \tau=t_{k}\end{cases}
$$

where

$$
\alpha_{2, \tau}=\left(\alpha_{2,0}+\frac{q}{2 a}\right) e^{2 a \tau}-\frac{q}{2 a}, \quad \tau \geq 0
$$

Together with centralized estimator (37), we apply the distributed estimator.

6.2.2. Distributed Fusion Estimate, $\widehat{z}_{t}^{\text {fus }}$. The distributed fusion equations for the example follow the same basic pattern as in Section 6.1.2. The local estimates $\widehat{x}_{\tau}^{(i)}=\mathbf{E}\left(x_{t} \mid y_{\left[t_{1}: t_{k}\right]}^{(i)}\right)$, corresponding error variances
$P_{\tau}^{(i i)}=\mathbf{E}\left(e_{t}^{(i)^{2}}\right)$, and cross-covariance $P_{\tau}^{(12)}=\mathbf{E}\left(e_{\tau}^{(1)} e_{\tau}^{(2)}\right)$ are described by the following:

Time update between measurements:

$$
\begin{aligned}
& \dot{\hat{x}}_{\tau}^{(i)^{-}}=a \widehat{x}_{\tau}^{(i)-}, \quad t_{k-1} \leq \tau \leq t_{k}, \quad \widehat{x}_{t_{k-1}}^{(i)^{-}}=\widehat{x}_{t_{k-1}}^{(i)}, \\
& \dot{P}_{\tau}^{(i i)^{-}}=2 a P_{\tau}^{(i i)-}+q, \quad P_{t_{k-1}}^{(i i)^{-}}=P_{t_{k-1}}^{(i i)}, \quad i=1,2, \\
& \dot{P}_{\tau}^{(12)-}=2 a P_{\tau}^{(12)-}+q, \quad t_{k-1} \leq \tau \leq t_{k}, \quad P_{t_{k-1}}^{(12)-}=P_{t_{k-1}}^{(12)},
\end{aligned}
$$

Measurement update at time $\tau=t_{k}$ :

$$
\begin{aligned}
& \hat{x}_{t_{k}}^{(i)}=\widehat{x}_{t_{k}}^{(i)-}+K_{t_{k}}^{(i)}\left(y_{t_{k}}^{(i)}-\widehat{x}_{t_{k}}^{(i)-}\right), \quad \hat{x}_{0}^{(i)-}=\bar{x}_{0}, \\
& K_{t_{k}}^{(i)}=\frac{P_{t_{k}}^{(i i)-}}{r_{i}+P_{t_{k}}^{(i i)-}}, \\
& P_{t_{k}}^{(i i)}=\left(1-K_{t_{k}}^{(i)}\right) P_{t_{k}}^{(i i)-}, \quad P_{0}^{(i i)-}=\sigma_{0}^{2}, \\
& P_{t_{k}}^{(12)}=\left(1-K_{t_{k}}^{(1)}\right)\left(1-K_{t_{k}}^{(2)}\right) P_{t_{k}}^{(12)-}, \quad P_{0}^{(12)-}=\sigma_{0}^{2} .
\end{aligned}
$$

The solution of (44a) and (44b) is given by

$$
\begin{aligned}
& P_{\tau}^{(i i)}= \begin{cases}P_{\tau}^{(i i)}, & t_{k-1} \leq \tau<t_{k} \\
P_{t_{k}}^{(i i)}, & \tau=t_{k}\end{cases} \\
& P_{\tau}^{(12)}= \begin{cases}P_{\tau}^{(12)}, & t_{k-1} \leq \tau<t_{k} \\
P_{t_{k}}^{(12)}, & \tau=t_{k},\end{cases}
\end{aligned}
$$

where

$$
P_{\tau}^{(i i)}=\left(P_{t_{k-1}}^{(i i)}+\frac{q}{2 a}\right) e^{2 a\left(\tau-t_{k-1}\right)}-\frac{q}{2 a}, \quad P_{t_{k}}^{(i i)}=\frac{r_{i} \sigma_{0}^{2}}{r_{i}+k \sigma_{0}^{2}},
$$$$
i=1,2 \text {, }
$$

$$
\begin{aligned}
& P_{\tau}^{(12)}=\left(P_{t_{k-1}}^{(12)}+\frac{q}{2 a}\right) e^{2 a\left(\tau-t_{k-1}\right)}-\frac{q}{2 a}, \\
& P_{t_{k}}^{(12)}=\frac{r_{1} r_{2} \sigma_{\theta}^{2}}{\left(r_{1}+k \sigma_{\theta}^{2}\right)\left(r_{2}+k \sigma_{\theta}^{2}\right)}, \\
& t_{k-1} \leq \tau<t_{k}, \quad i=1,2 ; \quad k=0,1,2, \ldots
\end{aligned}
$$

Next, two local estimates for the power of signal $z_{t}=x_{t}^{2}$ take the form $\widehat{z}_{\tau}^{(i)}=P_{\tau}^{(i i)}+\left(\widehat{x}_{\tau}^{(i)}\right)^{2}, i=1,2$. Combining $\widehat{z}_{\tau}^{(1)}$ and $\widehat{z}_{\tau}^{(2)}$ based on (11), we obtain the distributed fusion estimate

$$
\begin{array}{r}
\widehat{z}_{\tau}^{\text {fus }}=a_{\tau}^{(1)} \widehat{z}_{t_{k}}^{(1)}+a_{\tau}^{(2)} \widehat{z}_{\tau}^{(2)}, \quad a_{\tau}^{(1)}+a_{\tau}^{(2)}=1, \\
t_{k-1} \leq \tau \leq t_{k},
\end{array}
$$


where

$$
\begin{aligned}
& a_{\tau}^{(1)}=\frac{P_{z, \tau}^{(22)}-P_{z, \tau}^{(12)}}{P_{z, \tau}^{(11)}-2 P_{z, \tau}^{(12)}+P_{z, \tau}^{(22)}}, \\
& a_{\tau}^{(2)}=\frac{P_{z, \tau}^{(11)}-P_{z, \tau}^{(12)}}{P_{z, \tau}^{(11)}-2 P_{z, \tau}^{(12)}+P_{z, \tau}^{(22)}},
\end{aligned}
$$

with the covariance $P_{z, \tau}^{(i j)}$ which is calculated as

$$
\begin{gathered}
P_{z, \tau}^{(i i)}=\mathbf{E}\left[\left(x_{\tau}^{2}-\widehat{z}_{\tau}^{(i)}\right)^{2}\right]=4 S_{t} P_{\tau}^{(i i)}-2 P_{\tau}^{(i i)^{2}}+4 m_{\tau}^{2} P_{\tau}^{(i i)}, i=1,2, \\
P_{z, \tau}^{(12)}=\mathbf{E}\left[\left(x_{\tau}^{2}-\widehat{z}_{\tau}^{(1)}\right)\left(x_{\tau}^{2}-\widehat{z}_{\tau}^{(2)}\right)\right] \\
=4 m_{\tau}^{2} P_{\tau}^{(12)}+2 P_{\tau}^{(12)^{2}}+4 P_{\tau}^{(11)} P_{\tau}^{(22)} \\
+4 P_{\tau}^{(12)}\left(S_{\tau}-P_{\tau}^{(11)}-P_{\tau}^{(22)}\right), \\
t_{k-1} \leq \tau \leq t_{k},
\end{gathered}
$$

where

$$
\begin{aligned}
& m_{\tau}=\mathbf{E}\left(x_{\tau}\right)=m_{t_{k-1}} e^{a\left(\tau-t_{k-1}\right)}, \quad k=1,2, \ldots ; m_{0}=\bar{x}_{0}, \\
& S_{\tau}=\operatorname{Var}\left(x_{\tau}\right)=\left(S_{t_{k-1}}+\frac{q}{2 a}\right) e^{2 a\left(\tau-t_{k-1}\right)}-\frac{q}{2 a} \\
& S_{0}=\sigma_{0}^{2} .
\end{aligned}
$$

Finally, the overall MSE $P_{\tau}^{\text {fus }}$ of the fusion estimate $\widehat{z}_{\tau}^{\text {fus }}$ is evaluated as

$$
P_{\tau}^{\text {fus }}=\mathbf{E}\left[\left(x_{\tau}^{2}-\widehat{z}_{\tau}^{\text {fus }}\right)^{2}\right]= \begin{cases}P_{\tau}^{\text {fus }}, & t_{k-1} \leq \tau<t_{k}, \\ P_{t_{k}}^{\text {fus }}, & \tau=t_{k},\end{cases}
$$

where

$$
P_{\tau}^{\text {fus }}=\left(a_{\tau}^{(1)}\right)^{2} P_{z, \tau}^{(11)}+\left(a_{\tau}^{(2)}\right)^{2} P_{z, \tau}^{(22)}+2 a_{\tau}^{(1)} a_{\tau}^{(2)} P_{z, \tau}^{(12)}
$$

Here, the weights $a_{\tau}^{(i)}$ and cross-covariance $P_{z, \tau}^{(i j)}$ are determined by (46) and (47), respectively.

6.2.3. Comparative Analysis of Centralized and Distributed Estimators. The model parameters are subjected to $a=-2$, $q=10, r_{1}=0.2, r_{2}=0.3, x_{0} \sim \mathrm{N}(1,1), t_{k}-t_{k-1}=0.1$, $k=1,2, \ldots, 20$. Figure 2 illustrates the MSEs of the power of signals $P_{t}^{\mathrm{opt}}$ and $P_{t}^{\mathrm{fus}}$. As we can see in Figure 2, the centralized estimator $\widehat{z}_{t}^{\text {opt }}$ is better than the distributed one $\widehat{z}_{t}^{\text {fus }}$; that is, $P_{t}^{\text {opt }}<P_{t}^{\text {fus }}$. However, the difference between $P_{t}^{\text {opt }}$ and $P_{t}^{\text {fus }}$ is negligible. The relative error $\Delta_{t}=\left|\left(P_{t}^{\mathrm{fus}}-P_{t}^{\mathrm{opt}}\right) / P_{t}^{\mathrm{opt}}\right| 100 \%$ within the observation period $t_{k} \in[0 ; 2]$ is about $6 \%$. For this reason, the distributed estimator for NCFs is suitable for real implementation in multisensory systems.

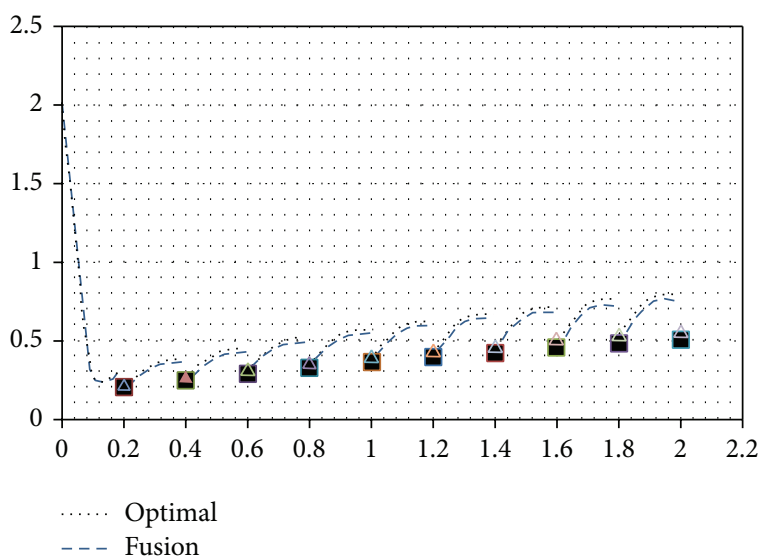

FIGURE 2: MSEs of fusion estimators for power of signal $z_{t}=x_{t}^{2}$.

\section{Application of Fusion Algorithms}

A comparative experimental analysis of the proposed estimators is considered for the motion of unmanned marine prober (UMP). In a marine inspection environment, UMP systems are often considered because they offer the benefits of convenience and human safety.

Assume a scenario in which the UMP detected an oiltanker accident, from which oil has spread out on a surface of the water without the influence of wind. As an initial action, the UMP estimates the length of a contour of the oil spread (Figure 3).

To control the size of a surface, the UMP needs to compute the distance from the oil tanker $d_{t}$ at every time instance representing an NCF

$$
d_{t}=f\left(x_{t}\right)=\sqrt{x_{1, t}^{2}+x_{2, t}^{2}}, \quad x_{t}=\left[\begin{array}{ll}
x_{1, t} & x_{2, t}
\end{array}\right]^{T},
$$

where $x_{1, t}$ and $x_{2, t}$ are coordinates of UMP.

Here, we verify the proposed fusion estimators using a linearized model of UMP [3]:

$$
\begin{gathered}
\dot{x}_{1, t}=x_{1, t}-2 x_{2, t}+v_{1, t}, \\
\dot{x}_{2, t}=x_{1, t}-x_{2, t}+v_{2, t},
\end{gathered}
$$

where $v_{t}^{(1)}$ and $v_{t}^{(2)}$ are uncorrelated zero-mean white Gaussian noises with intensities $q_{1}=q_{2}=0.1, t \in[0 ; 3], x_{1,0} \sim$ $\mathbf{N}(20 ; 0.2)$, and $x_{2,0} \sim \mathbf{N}(0 ; 0.2)$.

Next, with the help of systemic sensors such as ultrasonic sensors, sonar, radar, or GPS, the UMP measures the relative coordinates $x_{1, t}$ and $x_{2, t}$ from the oil tanker, respectively. Then, the measurement model for the UMP is given by

$$
y_{t}^{(1)}=x_{1, t}+w_{t}^{(1)}, \quad y_{t}^{(2)}=x_{2, t}+w_{t}^{(2)},
$$

where $w_{t}^{(1)}$ and $w_{t}^{(2)}$ are uncorrelated zero-mean white Gaussian sequences with intensities $r_{1}=r_{2}=0.1$.

Since the NCF is nonlinear, we apply the UT to calculate the local estimates $\widehat{z}_{t}^{(i)}$ and fusion estimates $\widehat{z}_{t}^{\text {opt }}$ and $\widehat{z}_{t}^{\text {fus }}$. The time update differential equations were solved by the 


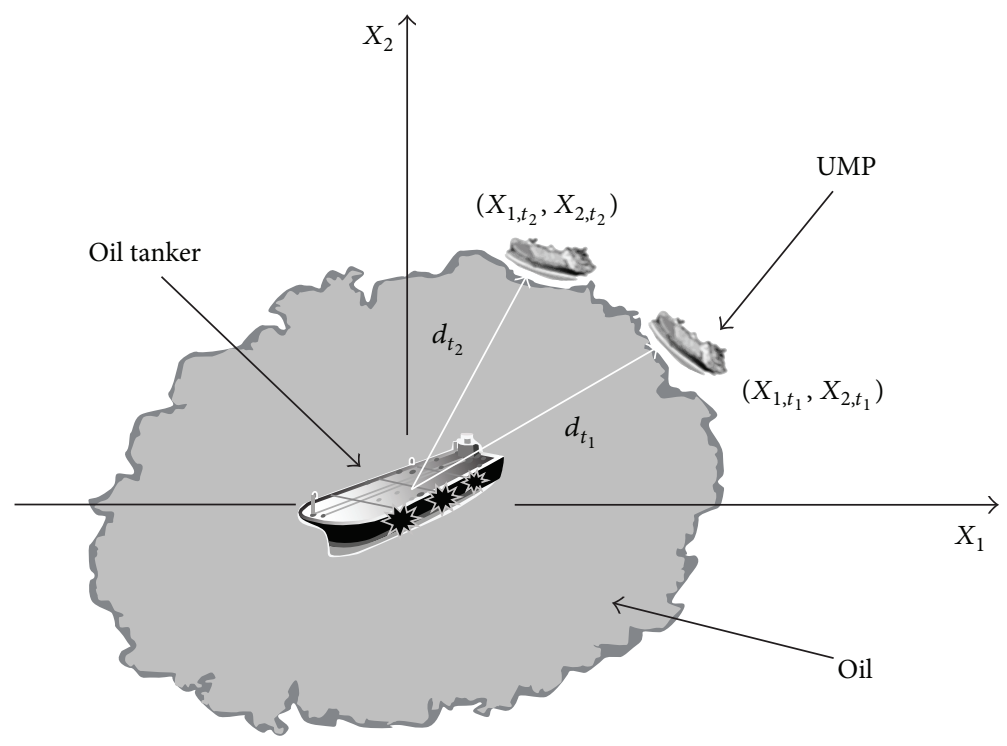

FIGURE 3: Estimation of size of oil spread contour.

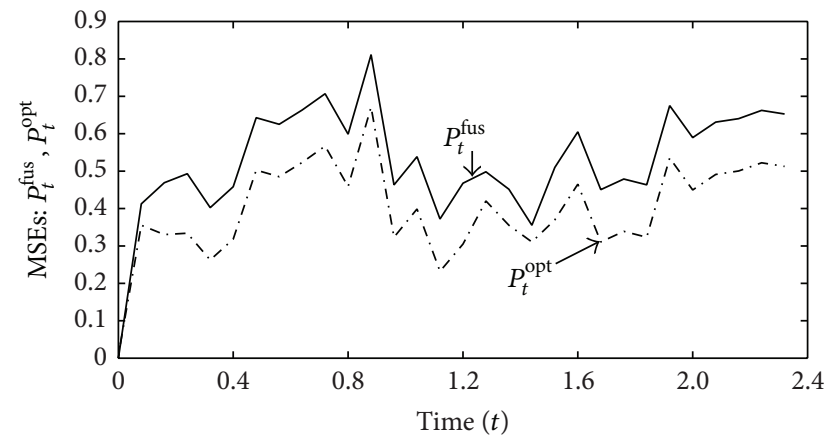

FIGURE 4: MSEs of fusion estimators for oil spread contour $d_{t}=$ $\sqrt{x_{1, t}^{2}+x_{2, t}^{2}}$.

Runge-Kutta scheme of the fourth order with the integration step $\Delta t=0.01$. To compare the MSEs $P_{t}^{\text {opt }}$ and $P_{t}^{\text {fus }}$, the Monte-Carlo method with 1000 runs was performed. Figure 4 illustrates the time histories of the MSEs for the both estimators.

As in Figure 4, the centralized estimate $\widehat{z}_{t}^{\text {opt }}$ has the best performance due to the lowest value of the MSE $P_{t}^{\text {opt }}<$ $P_{t}^{\text {fus }}$. As a result, we can confirm that we have verified that the decentralized estimator is more suitable for distributed processing in a multisensory environment.

\section{Conclusion}

In this paper, we derive a new centralized and decentralized estimator for nonlinear cost functions in mixed multisensor continuous-discrete stochastic systems. Computational approaches to their designing in practice are offered. Particular emphasis is given to a closed-form recursive procedure for a polynomial cost functions. The estimation accuracies of the proposed estimators are studied. In general, the centralized fusion estimator is considered as the most accurate, but, by the results of simulations with theoretical and real examples, the decentralized estimator demonstrates a reasonable accuracy. Furthermore, due to inherent drawbacks of centralized processing, the decentralized estimator may be more preferable in multisensory environment.

During the last decades, there has been extensive interest in the study of a class of physical systems modeled by hybrid system dynamics known as Markovian jump systems [2123]. As a generalization of the obtained results for mixed continuous-discrete stochastic systems, we would like to point out that it is possible to extend the main results to Markovian jump systems.

\section{Appendix}

The derivation of the equation for cross-covariance (13a) and cross-covariance (13b) is given as follows.

The Kalman equations (1) and (9a) and (9b) yield the linear differential difference equations for the local error $e_{\tau}^{(i)}=$ $x_{\tau}-\widehat{x}_{\tau}^{(i)}$

$$
\begin{gathered}
\dot{e}_{\tau}^{(i)-}=\dot{x}_{\tau}-\dot{\hat{x}}_{\tau}^{(i)}=F_{\tau} e_{\tau}^{(i)-}+G_{\tau} v_{\tau}, \quad t_{k-1} \leq \tau \leq t_{k} \\
e_{t_{k}}^{(i)}=x_{t_{k}}-\widehat{x}_{t_{k}}^{(i)} \\
=x_{t_{k}}-\widehat{x}_{t_{k}}^{(i)-}-K_{t_{k}}^{(i)}\left[H_{t_{k}}^{(i)} x_{t_{k}}+w_{t_{k}}^{(i)}-H_{t_{k}}^{(i)} \widehat{x}_{t_{k}}^{(i)-}\right] \\
=\left(I_{n}-K_{t_{k}}^{(i)} H_{t_{k}}^{(i)}\right) e_{t_{k}}^{(i)-}-K_{t_{k}}^{(i)} w_{t_{k}}^{(i)}, \\
e_{t_{k}}^{(i)-}=x_{t_{k}}-\widehat{x}_{t_{k}}^{(i)-} .
\end{gathered}
$$


Consequently,

$$
\begin{aligned}
\dot{P}_{\tau}^{(i j)-}= & \frac{d}{d \tau} \mathbf{E}\left[e_{\tau}^{(i)-}\left(e_{\tau}^{(j)-}\right)^{T}\right]=F_{\tau} \mathbf{E}\left[e_{\tau}^{(i)-}\left(e_{\tau}^{(j)-}\right)^{T}\right] \\
& +\mathbf{E}\left[e_{\tau}^{(i)-}\left(e_{\tau}^{(j)-}\right)^{T}\right] F_{\tau}^{T}+G_{\tau} Q_{\tau} G_{\tau}^{T} \\
= & F_{\tau} P_{\tau}^{(i j)-}+P_{\tau}^{(i j)-} F_{\tau}^{T}+G_{\tau} Q_{\tau} G_{\tau}^{T}, \\
P_{t_{k}}^{(i j)}= & \mathbf{E}\left[e_{t_{k}}^{(i)}\left(e_{t_{k}}^{(j)}\right)^{T}\right] \\
= & \mathbf{E}\left\{\left[\left(I_{n}-K_{t_{k}}^{(i)} H_{t_{k}}^{(i)}\right) e_{t_{k}}^{(i)-}-K_{t_{k}}^{(i)} w_{t_{k}}^{(i)}\right]\right. \\
& \left.\times\left[\left(I_{n}-K_{t_{k}}^{(j)} H_{t_{k}}^{(j)}\right) e_{t_{k}}^{(j)-}-K_{t_{k}}^{(j)} w_{t_{k}}^{(j)}\right]^{T}\right\} \\
= & \left(I_{n}-K_{t_{k}}^{(i)} H_{t_{k}}^{(i)}\right) \mathbf{E}\left[e_{t_{k}}^{(i)-}\left(e_{t_{k}}^{(j)-}\right)^{T}\right]\left(I_{n}-K_{t_{k}}^{(j)} H_{t_{k}}^{(j)}\right)^{T} \\
& -\left(I_{n}-K_{t_{k}}^{(i)} H_{t_{k}}^{(i)}\right) \mathbf{E}\left(e_{t_{k}}^{(i)-} w_{t_{k}}^{(j)^{T}}\right) K_{t_{k}}^{(j)^{T}} \\
& -K_{t_{k}}^{(i)} \mathbf{E}\left[w_{t_{k}}^{(i)}\left(e_{t_{k}}^{(j)-}\right)^{T}\right]\left(I_{n}-K_{t_{k}}^{(j)} H_{t_{k}}^{(j)}\right)^{T} \\
& +K_{t_{k}}^{(i)} \mathbf{E}\left(w_{t_{k}}^{(i)} w_{t_{k}}^{(j)^{T}}\right) K_{t_{k}}^{(j)^{T}}, i \neq j .
\end{aligned}
$$

Taking into account that $e_{t_{k}}^{(i)-}$ and $e_{t_{k}}^{(j)-}$ do not depend on measurements $y_{t_{k}}^{(j)}$ and $y_{t_{k}}^{(i)}$, respectively, and white noises $w_{t_{k}}^{(i)}$ and $w_{t_{k}}^{(j)}$ are uncorrelated at $i \neq j$, (A.3) yields linear recursive (13a) and (13b) for $P_{t_{k}}^{(i j)}$.

This completes the derivation of (13a) and (13b).

\section{Conflict of Interests}

The authors declare that there is no conflict of interests regarding the publication of this paper.

\section{Acknowledgments}

This work was supported by the Incheon National University Research Grant in 2014-2015 and in part by the National Research Foundation of Korea of the Ministry of Education, Science and Technology under Grant no. NRF2012R1A1A2000679.

\section{References}

[1] Y. Zhu, J. Zhou, X. Shen, E. Song, and Y. Luo, Networked Multisensor Decision and Estimation Fusion, CRC Press, Boca Raton, Fla, USA, 2013.

[2] M. E. Liggins, D. L. Hall, and J. Llinas, Eds., Handbook of Multisensor Data Fusion: Theory and Practice, The Electrical Engineering and Applied Signal Processing Series, CRC Press, Boca Raton, Fla, USA, 2nd edition, 2009.

[3] A. G. O. Mutambara, Decentralized Estimation and Control for Multisensor Systems, CRC Press, Boca Raton, Fla, USA, 1998.
[4] V. Shin, Y. Lee, and T.-S. Choi, "Generalized Millman's formula and its application for estimation problems," Signal Processing, vol. 86, no. 2, pp. 257-266, 2006.

[5] V. Shin, G. Shevlyakov, and K. Kim, "A new fusion formula and its application to continuous-time linear systems with multisensor environment," Computational Statistics and Data Analysis, vol. 52, no. 2, pp. 840-854, 2007.

[6] S.-L. Sun and Z.-L. Deng, "Multi-sensor information fusion kalman filter weighted by scalars for systems with colored measurement noises," Journal of Dynamic Systems, Measurement and Control, vol. 127, no. 4, pp. 663-667, 2005.

[7] J. Zhou, Y. Zhu, Z. You, and E. Song, "An efficient algorithm for optimal linear estimation fusion in distributed multisensor systems," IEEE Transactions on Systems, Man, and Cybernetics, vol. 36, pp. 1000-1009, 2006.

[8] S. H. Lee, D. Y. Kim, N.-V. Nguyen, and V. Shin, "Comparison of multi-sensor fusion filterss weighted by scalars and matrices," in Proceedings of the International Conference on Control, Automation and Systems (ICCAS '07), pp. 155-160, Seoul, Republic of Korea, October 2007.

[9] Z. Duan and X. R. Li, "Lossless linear transformation of sensor data for distributed estimation fusion," IEEE Transactions on Signal Processing, vol. 59, no. 1, pp. 362-372, 2011.

[10] S. Julier, J. Uhlmann, and H. F. Durrant-Whyte, "A new method for the nonlinear transformation of means and covariances in filters and estimators," IEEE Transactions on Automatic Control, vol. 45, no. 3, pp. 477-482, 2000.

[11] S. J. Julier and J. K. Uhlmann, "Unscented filtering and nonlinear estimation," Proceedings of the IEEE, vol. 92, no. 3, pp. 401422, 2004.

[12] D.-J. Lee, "Nonlinear estimation and multiple sensor fusion using unscented information filtering," IEEE Signal Processing Letters, vol. 15, pp. 861-864, 2008.

[13] B. Noack, D. Lyons, M. Nagel, and U. D. Hanebeck, "Nonlinear information filtering for distributed multisensor data fusion," in Proceedings of the American Control Conference (ACC '11), pp. 4846-4852, San Francisco, Calif, USA, July 2011.

[14] F. Gustafsson and G. Hendeby, "Some relations between extended and unscented Kalman filters," IEEE Transactions on Signal Processing, vol. 60, no. 2, pp. 545-555, 2012.

[15] F. L. Lewis, Optimal Estimation: With an Introduction to Stochastic Control Theory, John Wiley \& Sons, New York, NY, USA, 1986.

[16] A. Gelb, Applied Optimal Estimation, MIT Press, Cambridge, Mass, USA, 1974.

[17] V. Pugachev and I. Sinitsyn, Stochastic Differential Systems: Analysis and Filtering, John Wiley \& Sons, Chichester, UK, 1987.

[18] R. Kan, "From moments of sum to moments of product," Journal of Multivariate Analysis, vol. 99, no. 3, pp. 542-554, 2008.

[19] B. Holmquist, "Expectations of products of quadratic forms in normal variables," Stochastic Analysis and Applications, vol. 14, no. 2, pp. 149-164, 1996.

[20] L. Chang, B. Hu, A. Li, and F. Qin, "Transformed unscented Kalman filter," IEEE Transactions on Automatic Control, vol. 58, no. 1, pp. 252-257, 2013.

[21] Z.-G. Wu, P. Shi, H. Su, and J. Chu, "Passivity analysis for discrete-time stochastic markovian jump neural networks with mixed time delays," IEEE Transactions on Neural Networks, vol. 22, no. 10, pp. 1566-1575, 2011. 
[22] Zh. Wu, P. Shi, H. Su, and J. Chu, "Asynchronous $l_{2}-l_{\infty}$ filtering for discrete-time stochastic Markov jump systems with randomly occurred sensor nonlinearities," Automatica, vol. 50, no. 1, pp. 180-186, 2014.

[23] H. Shen, S. Xu, J. Lu, and J. Zhou, "Passivity-based control for uncertain stochastic jumping systems with mode-dependent round-trip time delays," Journal of the Franklin Institute, vol. 349, no. 5, pp. 1665-1680, 2012. 


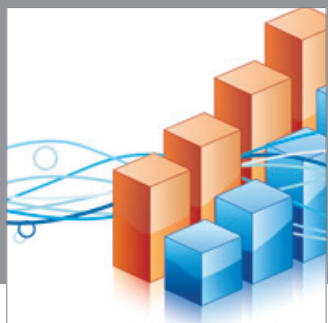

Advances in

Operations Research

mansans

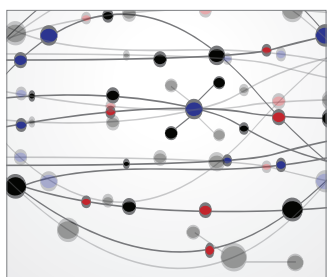

The Scientific World Journal
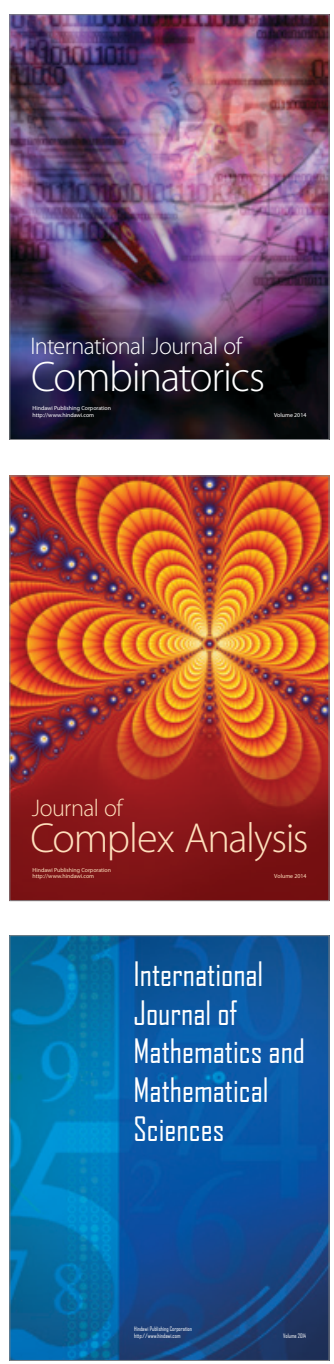
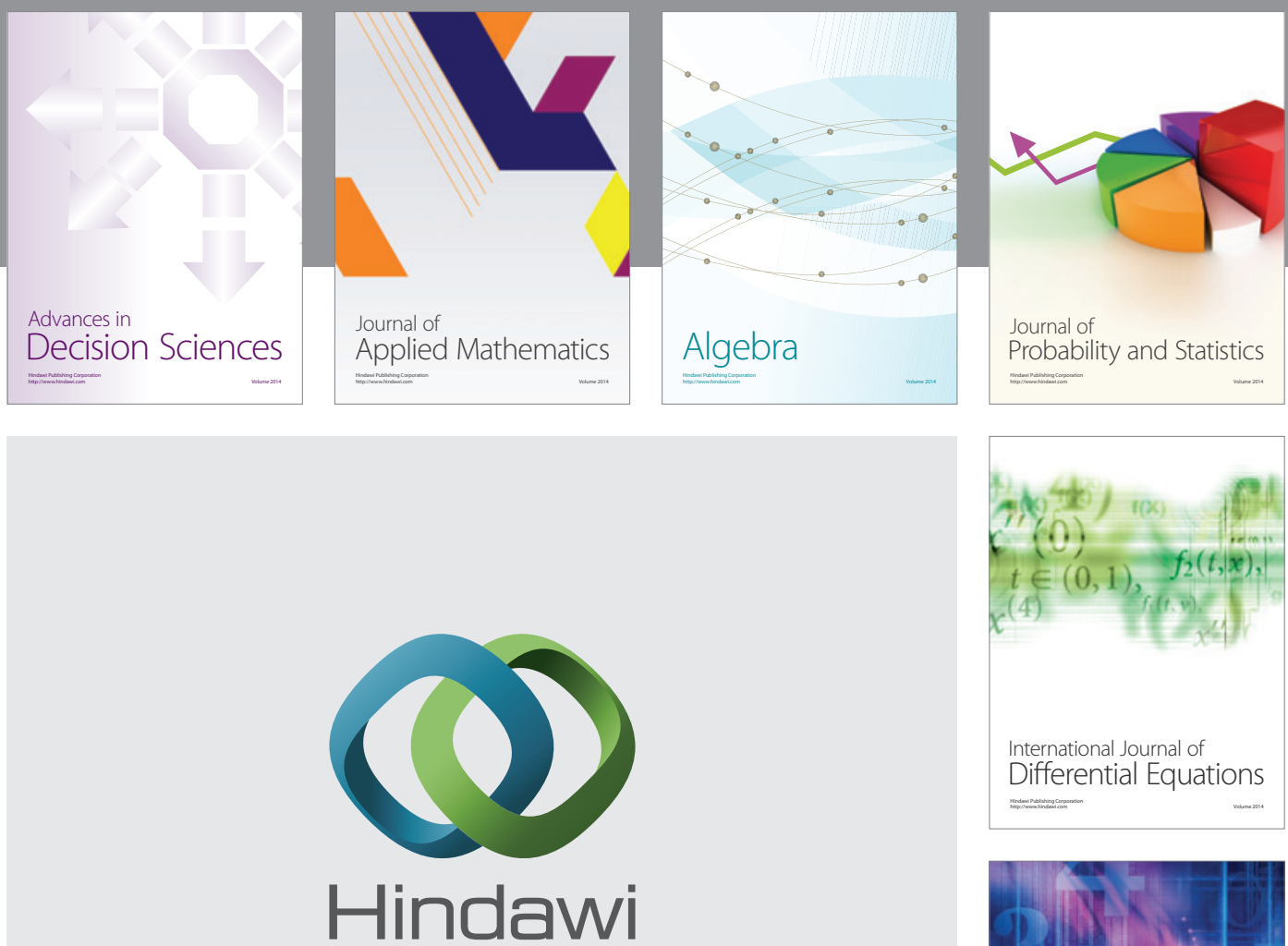

Submit your manuscripts at http://www.hindawi.com
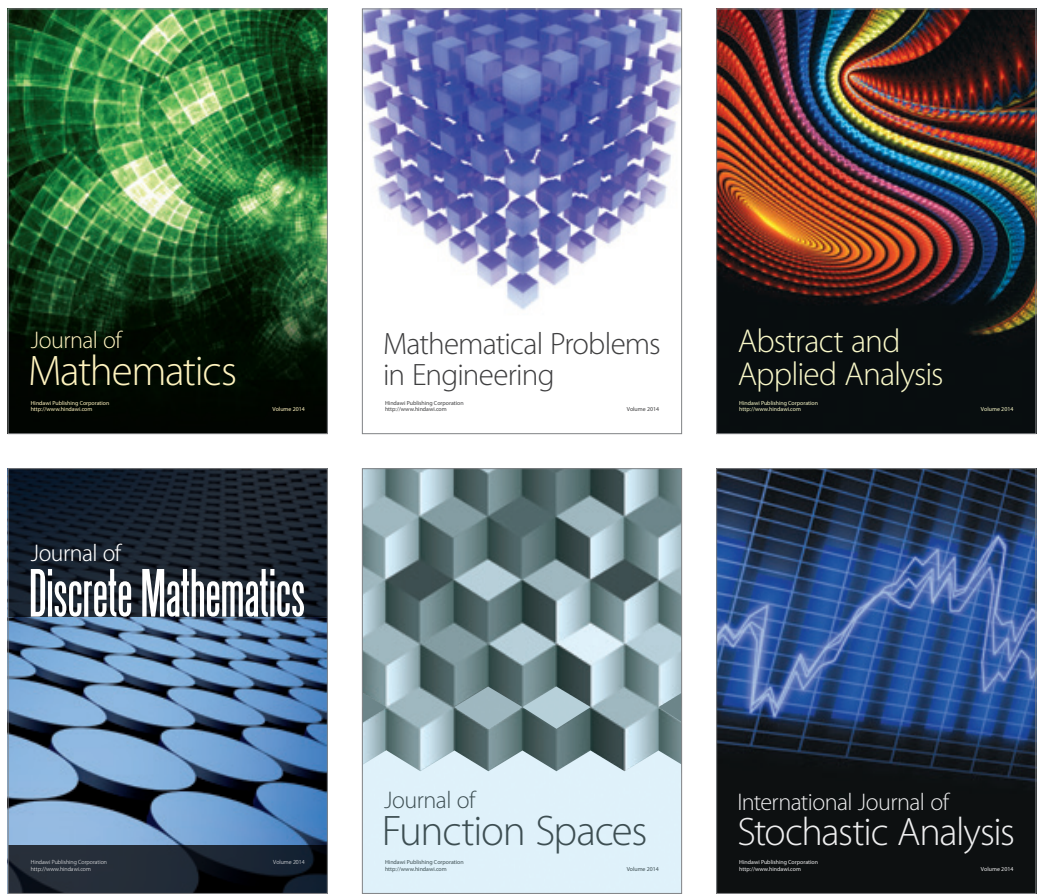

Journal of

Function Spaces

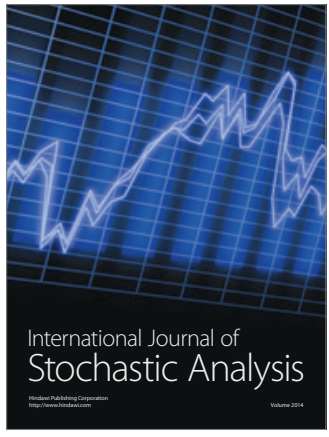

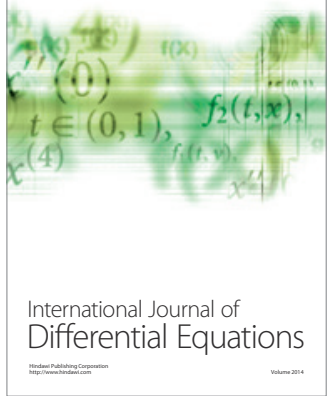
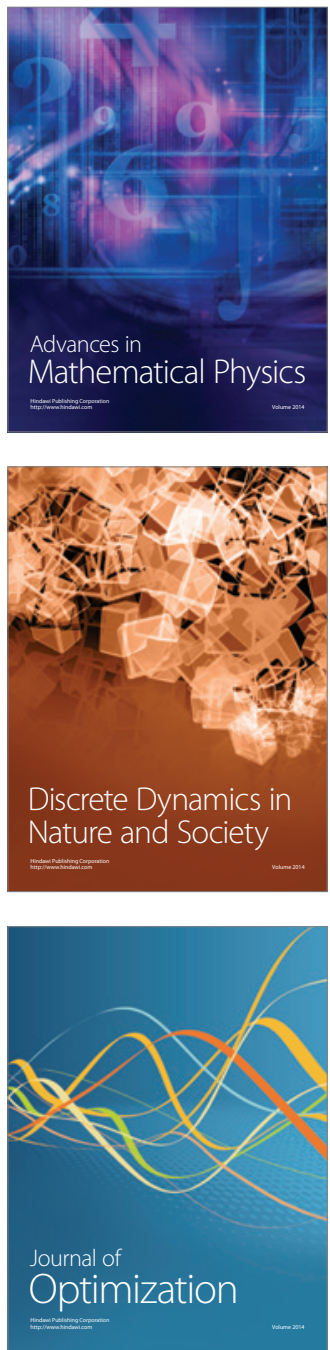